\title{
Genomic content of chemosensory receptors in two sister blister beetles facilitates characterization of chemosensory evolution
}

\author{
Yuan-Ming $\mathrm{Wu}^{1,2}$, Yang-Yang Liu ${ }^{3}$ and Xiang-Sheng Chen ${ }^{3 *}$
}

\begin{abstract}
Background: More than 2500 species belong to the Meloidae family (Coleoptera: Tenebrionoidea), members of which produce the potent defensive blistering agent cantharidin and are commonly known as blister beetles or Spanishflies. Cantharidin has recently been used for cancer therapy. Hycleus cichorii and Hycleus phaleratus have been used in traditional Chinese medicine for more than 2000 years due to their ability to biosynthesize cantharidin. To understand the role of the chemosensory system in beetle evolution, we comparatively analysed the chemosensory receptor families of both blister beetle species and compared them with those of other beetles.

Results: We identified 89 odorant receptors (ORs), 86 gustatory receptors (GRs), and 45 ionotropic receptors (IRs) in H. phaleratus and 149 ORs, 102 GRs and 50 IRs in H. cichorii. Nine groups of beetle ORs were recovered, and a similar pattern of ORs in Coleoptera emerged. Two evident expanded clades in Hycleus (Groups 5A and 3) were reconstructed in the phylogenetic tree. Four of eight genes with evidence of positive selection were clustered in the expanded clades of Group 5A. Three, eight and three orthologous pairs of $\mathrm{CO}_{2}$, sugar and fructose receptors, respectively, were identified in both blister beetles. Two evident expanded clades of putative bitter GRs in Hycleus were also found, and the GR in one clade had notably low divergence. Interestingly, IR41a was specifically expanded in blister beetles compared to other insects identified to date, and IR75 was also clearly expanded in both blister beetles based on our phylogenetic tree analysis. Moreover, evidence of positive selection was detected for eight ORs, three GRs and two IRs, half of which were from five duplicate clades.
\end{abstract}

Conclusions: We first annotated the chemosensory receptor families in a pair of sister beetle genomes (Meloidae: Hycleus), which facilitated evolutionary analysis of chemosensory receptors between sibling species in the Coleoptera group. Our analysis suggests that changes in chemosensory receptors have a possible role in chemicalbased species evolution in blister beetles. Future studies should include more species to verify this correlation, which will help us understand the evolution of blister beetles.

Keywords: Blister beetle (Spanishfly), Chemosensory, Gustatory receptor, Odorant receptor, Ionotropic receptors

\footnotetext{
*Correspondence: xschen@gzu.edu.cn

${ }^{3}$ Institute of Entomology/Special Key Laboratory for Development and

Utilization of Insect Resources, Guizhou University, Guiyang, Guizhou, P.R.

China 550025

Full list of author information is available at the end of the article
}

(c) The Author(s). 2020 Open Access This article is licensed under a Creative Commons Attribution 4.0 International License, which permits use, sharing, adaptation, distribution and reproduction in any medium or format, as long as you give appropriate credit to the original author(s) and the source, provide a link to the Creative Commons licence, and indicate if changes were made. The images or other third party material in this article are included in the article's Creative Commons licence, unless indicated otherwise in a credit line to the material. If material is not included in the article's Creative Commons licence and your intended use is not permitted by statutory regulation or exceeds the permitted use, you will need to obtain permission directly from the copyright holder. To view a copy of this licence, visit http://creativecommons.org/licenses/by/4.0/ The Creative Commons Public Domain Dedication waiver (http://creativecommons.org/publicdomain/zero/1.0/) applies to the data made available in this article, unless otherwise stated in a credit line to the data. 


\section{Background}

To adapt to varied environments and ecological niches, insects rely upon their sensory system to distinguish chemical signals, such as pheromones and plant volatiles. An understanding of the relative changes in genes among closely related insect species is required to help elucidate the role of chemosensory adaptation in different environments because the chemical-sensing system is important for host and mate recognition [1]. The evolutionary events of gene duplication, amino acid mutation and gene expression variation may play essential roles during speciation or adaptive evolution. For example, during the process of host specialization of Drosophila sechellia, rapid evolution events and expression divergence were widely detected in chemosensory genes, such as gustatory receptor (GR) genes, odorant receptor (OR) genes and genes encoding odorant-binding proteins (OBPs) [2-4]; certain chemoreceptor genes with mutations might play key roles in local adaptation and reproductive isolation in the pea aphid Acyrthosiphon pisum [5]; in Pyrrhalta beetles, protein mutations and changes in the expression of some particular chemosensory genes are associated with reproductive isolation and host shift [6]; and the changes in the expression of genes encoding particular proteins (OBPs) and ORs are associated with the host plant shift in Chrysomela lapponica [7].

In insects, at least three chemosensory receptor families are involved in the recognition of chemical signals. OR gene families are usually expressed in olfactory sensory neurons and are involved in the detection of volatile chemicals [8-12]. GRs are mainly involved in the detection of contact chemicals or carbon dioxide [13-15], and ionotropic receptors (IRs) are known to recognize acids, aromatics and nitrogen-containing compounds [16, 17]. These genes allow insects to seek and select hosts and mates and thus can affect adaptive changes in closely related species.

More than 2500 species of insects commonly known as blister beetles or Spanishflies belong to the Meloidae family, and more than 1500 of these beetle species are known to produce cantharidin [18]. Cantharidin $\left(\mathrm{C}_{10} \mathrm{H}_{12} \mathrm{O}_{4}\right)$ is widely used in anti-insect and bacterial products in agricultural and medical applications worldwide [18-20]. Recently, cantharidin and its derivatives have been used to treat several cancers, including stomach, liver, lung and oesophageal cancers [21-23]. The dried body of this beetle has been used as a traditional medicine in China for the past 2000 years. However, only Hycleus cichorii Linnaeus and Hycleus phaleratus Pallas are widely used and listed in the Pharmacopoeia of the People's Republic of China [24].

These two beetles have largely overlapping sympatric ranges in China and a similar emergence phenology and appearance, except that $H$. phaleratus has a larger body size than $H$. cichorii. These beetles are mainly distributed in Southwest China and feed on the eggs of Locustidae species during the larval period and on legumes and cucurbitaceous crops in adulthood. However, in our field survey experience, the $H$. phaleratus population has declined faster than the $H$. cichorii population in recent years due to destruction of their habitats by human activity, including capture by humans. Therefore, an understanding of the changes that underlie speciation between these two sister species and comparison of the chemosensory proteins that are highly important for insect survival and reproduction and represent interesting strategies for species evolution can provide important insights. Although several studies have compared differences in chemosensory genes between sister species [3$7,25,26]$, most of these studies have investigated this aspect partly by RNA-seq, and relatively few studies have comprehensively examined how sister taxa differ in this aspect, especially in Coleoptera groups. In this study, we identified the members of three receptor families involved in chemosensory perception in a pair of sister blister beetles and analysed the evolution of these receptors in beetles.

\section{Results}

The annotated candidate chemosensory receptors were identified by tBLASTn and manual verification. In total, we identified 89 ORs, 86 GRs and 45 IRs in $H$. phaleratus and 149 ORs, 102 GRs and 50 IRs in H. cichorii (Table 1, Additional file 1 and Additional file 2). Moreover, 12 and 14 ionotropic glutamate receptors (iGluRs; five full-length genes in each) were identified in $H$. phaleratus and $H$. cichorii, respectively (Table 1, Additional file 1 and Additional file 2). All the coding sequences (CDSs) and proteins identified in this study are listed in Additional file 1, and all the gene locations and exon boundaries are listed in Additional file 2.

\section{Odorant receptors}

We identified 89 (including 44 full-length and 20 pseudogenes) and 149 (including 77 full-length and 30 pseudogenes) ORs in $H$. phaleratus and $H$. cichorii, respectively. In both species, the numbers of introns in the full-length OR genes vary between two and six, with more than $90 \%$ ranging from three to five. Additionally, HcicORco and HphaORco were also identified (Fig. 1). Recently, the coleopteran OR subfamilies were reclassified by Mitchell et al. [27]. Based on Mitchell's study, nine beetle OR groups were recovered in our data (Fig. 1). The blister beetle ORs are distributed in seven groups, lacking Group 4 and 5B, indicating loss of ORs from these two groups. Most Hycleus ORs appeared in Groups 5A, 3 and 2A (Fig. 1). Most ORs of 
Table 1 Number of Chemosensory Genes in the Genome of the Two Sister Blister Beetles H. phaleratus and H. cichorii. The numbers in parentheses are the gene numbers from the phylogenetic tree, the full-length genes and pseudogenes

\begin{tabular}{llll}
\hline Species & ORs & GRs & IRs/iGluRs \\
\hline H. phaleratus & $\mathbf{8 9 ( 7 2 / 4 4 / 2 0 )}$ & $\mathbf{8 6 ( 7 4 / 6 8 / 9 )}$ & $\mathbf{4 5 ( 4 5 / 2 5 / 6 ) / 1 2 ( 1 2 / 5 / 1 )}$ \\
H. cichorii & $149(130 / 77 / 30)$ & $102(87 / 62 / 19)$ & $50(50 / 30 / 8) / 14(14 / 5 / 1)$ \\
Orthologous pairs & $53[8]$ & $55[3]$ & $39[2] / 11[1]$ \\
[positively select] & & &
\end{tabular}

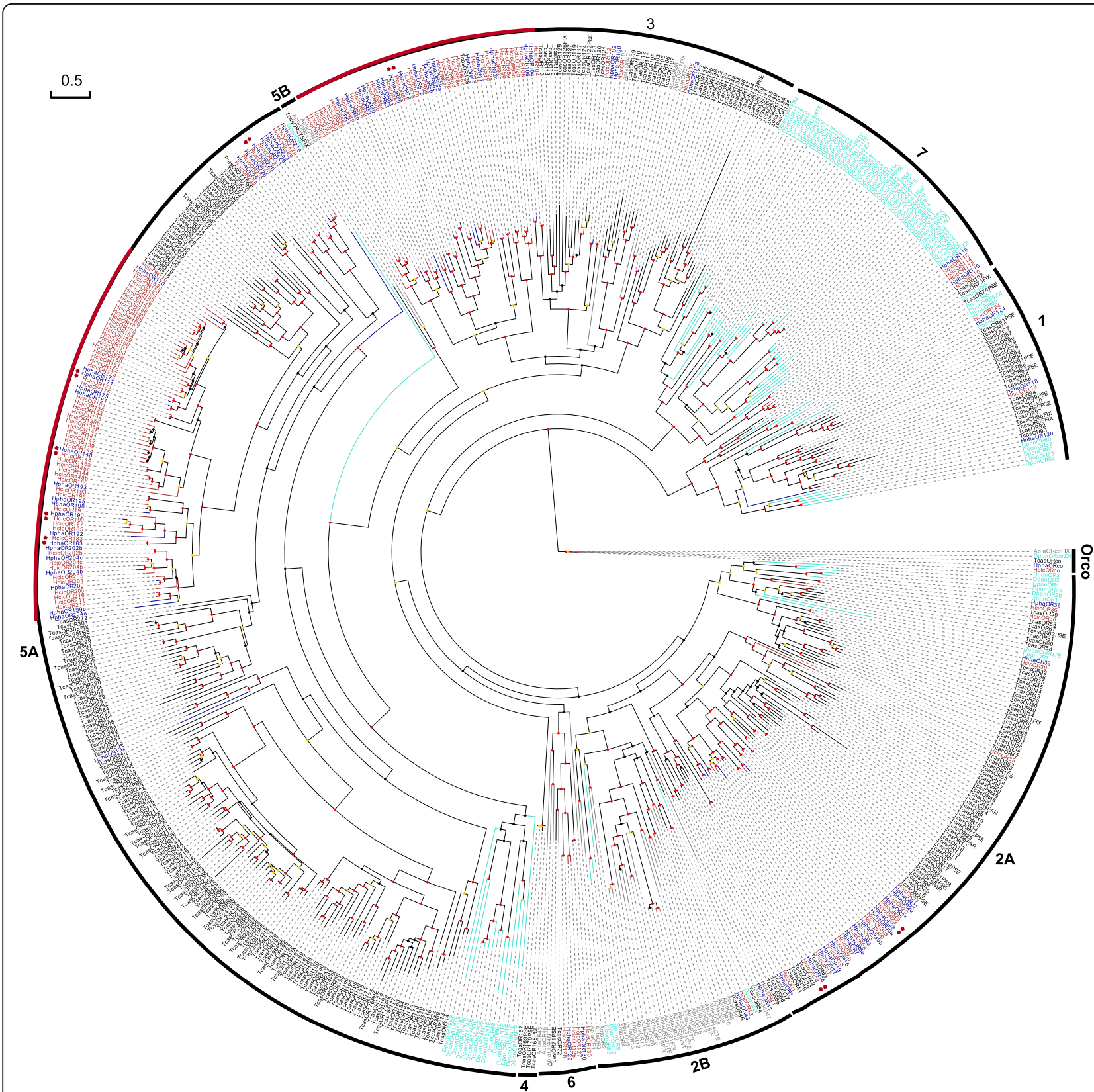

Fig. 1 Maximum Likelihood Phylogeny Based on the Protein Sequences of the Candidate ORs. Red: H. cichorii (Hcic); blue: H. phaleratus (Hpha); black: T. castaneum (Tcas); turquoise: D. ponderosae (Dpon); and grey: A. planipennis (Apla). The tree is based on MAFFT alignment, was constructed using RAxML, and was rooted with the conserved lineage of Orco proteins. The coloured dots indicate nonparametric bootstrap support (\%): red: $\geq 75$, yellow: 50-74, and black: $<50$. Two major Hycleus expanded clades are evident (red arc). The red asterisk $(*)$ marks the positively selected gene pairs 
Dendroctonus ponderosae were classified in Group 7, whereas the largest expansion in Agrilus planipennis was observed in Group 2B (Fig. 1), which is consistent with previous reports [28]. Notably, two evident expanded OR clades were identified based on our phylogenetic tree (Fig. 1, red arc in Groups 3 and 5A), containing more than $50 \%$ of the Hycleus ORs. Although up to $67 \%$ more ORs were identified in $\mathrm{H}$. cichorii than in its sister species (149 vs 89 ORs), nearly all these additional $H$. cichorii ORs were clustered in two clades. The ORs in the other clades were almost all single-copy (1:1) orthologues in blister beetles. Moreover, we identified 53 pairs of orthologues between the two beetle species based on the maximum likelihood (ML) tree, where 8 pairs of orthologues showed evidence of positive selection (Table 1, Fig. 1 and Additional file 3). Among these pairs, a total of 5 pairs, 2 pairs and 1 pair were distributed in Groups 5A, 2A and 3, respectively. Interestingly, four pairs of genes with evidence of positive selection were clustered in the expanded clades of Group 5A (Fig. 1).

\section{Gustatory receptors}

We identified 86 (including 68 full-length genes and 9 pseudogenes) and 102 (including 62 full-length genes and 19 pseudogenes) GRs in $H$. phaleratus and $H$. cichorii, respectively. Three pairs of $\mathrm{CO}_{2}$ receptors were identified (named GR1-3), containing two introns, five introns and one intron, respectively, and this group presented 1:1 orthologues among these beetles in our phylogenetic tree (Fig. 2). Eight pairs of conserved sugar receptors were also detected in this study (named GR411; Fig. 2), containing four to six introns. Additionally, three pairs of homologues and an additional paralogue $H$. cichorii GR, which functions as a fructose receptor, were detected, containing two to five introns. The flour beetle was annotated with the most (eight) copies of the fructose receptor in these six analysed beetle species, followed by the blister beetles, and the remaining three beetles had only one copy. The remaining GRs are putative bitter GRs, and most full-length bitter GRs contain one or two introns (with a total range of one to five), similar to those in D. ponderosae and A. planipennis [28]. Almost all bitter GRs from the other four species were grouped into small to large species-specific expanded clades. Two blister beetle expanded clades emerged in our phylogenetic tree, which included 44\% bitter GRs (red arc in Fig. 2). Similar to the expanded clades of ORs, these GRs in one clade showed notably low divergence among each other. The bitter GRs in the Hycleus genus exhibit high conservation, and more than 55 pairs of orthologues were shared by the two blister beetle species, with more than $2 / 3$ of the $H$. phaleratus GRs existing in orthologue pairs (Fig. 2). A total of 3 pairs of orthologues between the blister beetle species showed evidence of positive selection, and one pair (GR34) was located in an expanded clade (Table 1, Fig. 2 and Additional file 3).

\section{Ionotropic receptors}

IRs are closely related to iGluRs [29, 30]. A total of 45 and 50 IRs (including 6 and 8 pseudogenes, respectively) and 12 and 14 iGluRs (1 pseudogene in each) were identified in $H$. phaleratus and $H$. cichorii, respectively (Table 1). IR25a and IR8a are considered coreceptor IRs, which are highly conserved in beetles, and a 1:1 orthologue was present in our ML tree (Fig. 3). The conserved antennal IRs, including IR75, IR8, IR25 IR40a, IR21a, IR68a, IR60a, IR41a, IR76b and IR93a, were identified in blister beetles (Fig. 3 and Table 2). Seven of these ten clades contained only one copy in both blister beetle species, and five were present as 1:1 orthologues in all the analysed beetles (Fig. 3 and Table 2). Notably, a total of 10 (including one pseudogene) and 13 (including three pseudogenes) copies of IR41a were annotated in $H$. phaleratus and $H$. cichorii, respectively. However, the corresponding IRs in other species had no more than 3 copies (Fig. 3 and Table 2). IR41a was clearly expanded in blister beetles compared to other beetles. IR41a is not a unique example; for example, IR75 was expanded in blister beetle species, with 8 (including one pseudogene) and 13 (including three pseudogenes) copies in these two beetles, and the gene number of this clade was more variable than that of the other antennal IRs (Fig. 3 and Table 2). The remaining putative divergent IRs (18 and 16 in $H$. phaleratus and $H$. cichorii, respectively) almost all appeared as pairs in the phylogenetic tree. Two copies of IR100 were also identified in both species. The IRs showed high conservation between these two beetles. A total of 39 pairs of orthologous IRs were found between the two species, and 2 pairs of orthologues showed evidence of positive selection, namely, IR41a2-1 and IR112 (Table 1, Fig. 3 and Additional file 3).

Chemosensory receptors are often present as tandem repeat clusters in the genome and are therefore more difficult to assemble than other genomic regions, which may represent the largest obstacle to annotating all members of such gene families. The current genomes of these blister beetles were assembled from nextgeneration sequencing (NGS) data, and the scaffold N50 length was only $79 \mathrm{~kb}$ in $H$. cichorii and $56 \mathrm{~kb}$ in $H$. phaleratus; moreover, Benchmarking Universal Single-Copy Orthologs (BUSCO) evaluation showed $\sim 92 \%$ complete BUSCO in both species [33]. To verify whether some putative receptors were not assembled in the genome, we de novo assembled RNA-seq data and mapped these sequences onto the known corresponding protein and genome sequences. The results showed that all receptor 


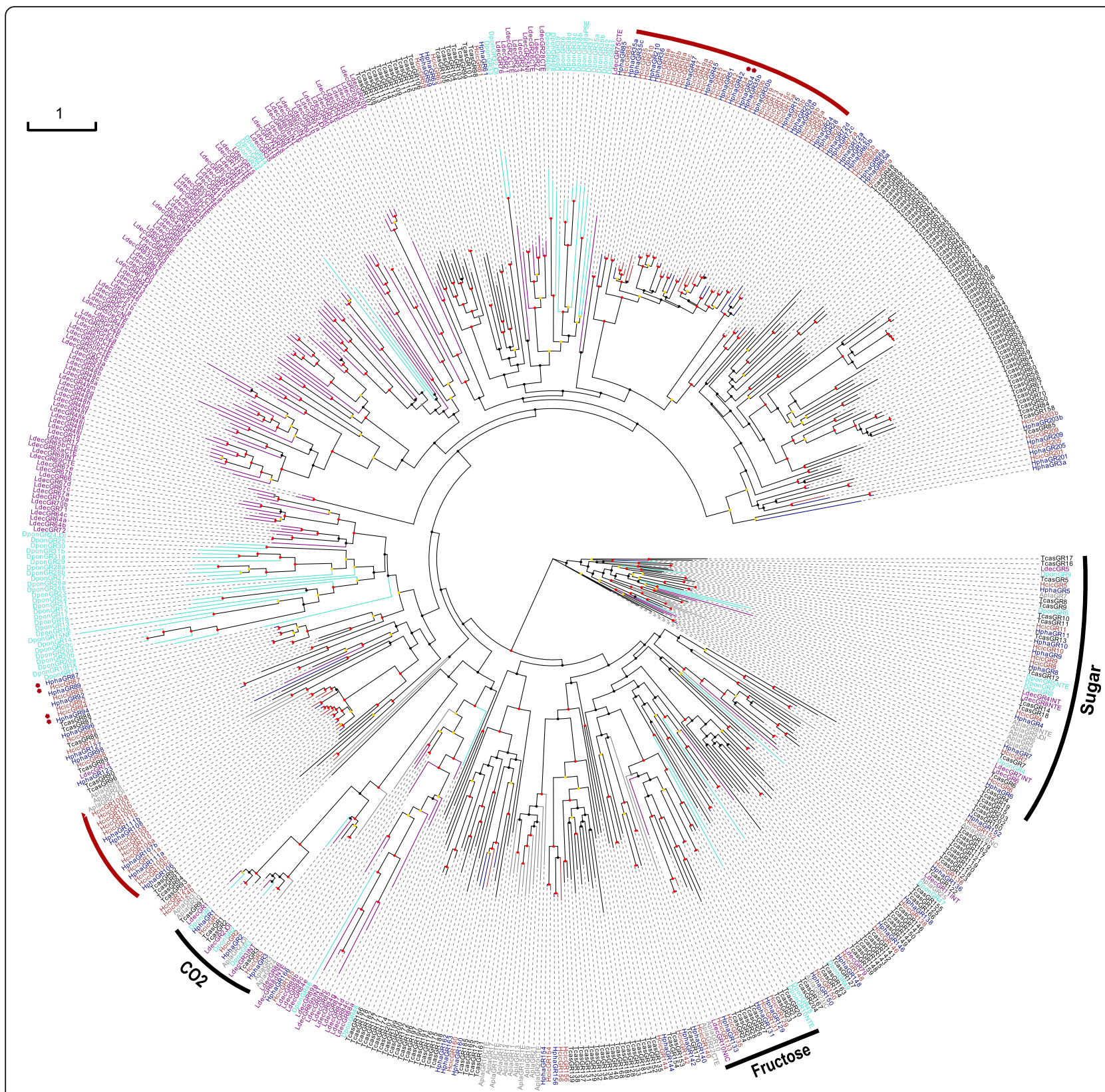

Fig. 2 Maximum Likelihood Phylogeny Based on the Protein Sequences of the Candidate GRs. Red: H. cichorii (Hcic); blue: H. phaleratus (Hpha); black: T. castaneum (Tcas); turquoise: D. ponderosae (Dpon); purple: L. decemlineata (Ldec) and grey: A. planipennis (Apla). The tree is based on MAFFT alignment, was constructed using RAxML, and was rooted with the conserved lineage of putative sugar receptors. The coloured dots indicate nonparametric bootstrap support (\%): red: $\geq 75$, yellow: 50-74, and grey: <50. Two recent Hycleus expanded clades are evident (red arc). The red asterisk $\left(^{*}\right)$ marks the positively selected gene pairs

genes identified via RNA-seq were also annotated in the H. cichorii genome (Fig. 4 and Table 1). Few GRs and IRs were missed in the $H$. phaleratus genome, while $17.4-30.0 \%$ of the ORs identified via RNA-seq were missing from this genome (Fig. 4 and Table 1). In $H$. cichorii, all receptor genes identified via RNA-seq were annotated in the genome (Fig. 4), which may be beneficial for evolutionary analyses of these three gene families in blister beetle within Coleoptera.

\section{Discussion}

We first identified the chemosensory receptor families at the whole-genome level in a pair of sister beetle species (Coleoptera: Meloidae) and performed an evolutionary analysis of chemosensory receptors within Coleoptera groups. In Coleoptera, surprisingly few studies have examined how sister taxa differ in chemosensory gene evolution, although two leaf beetles (Pyrrhalta aenescens and Pyrrhalta maculicollis) [6] 


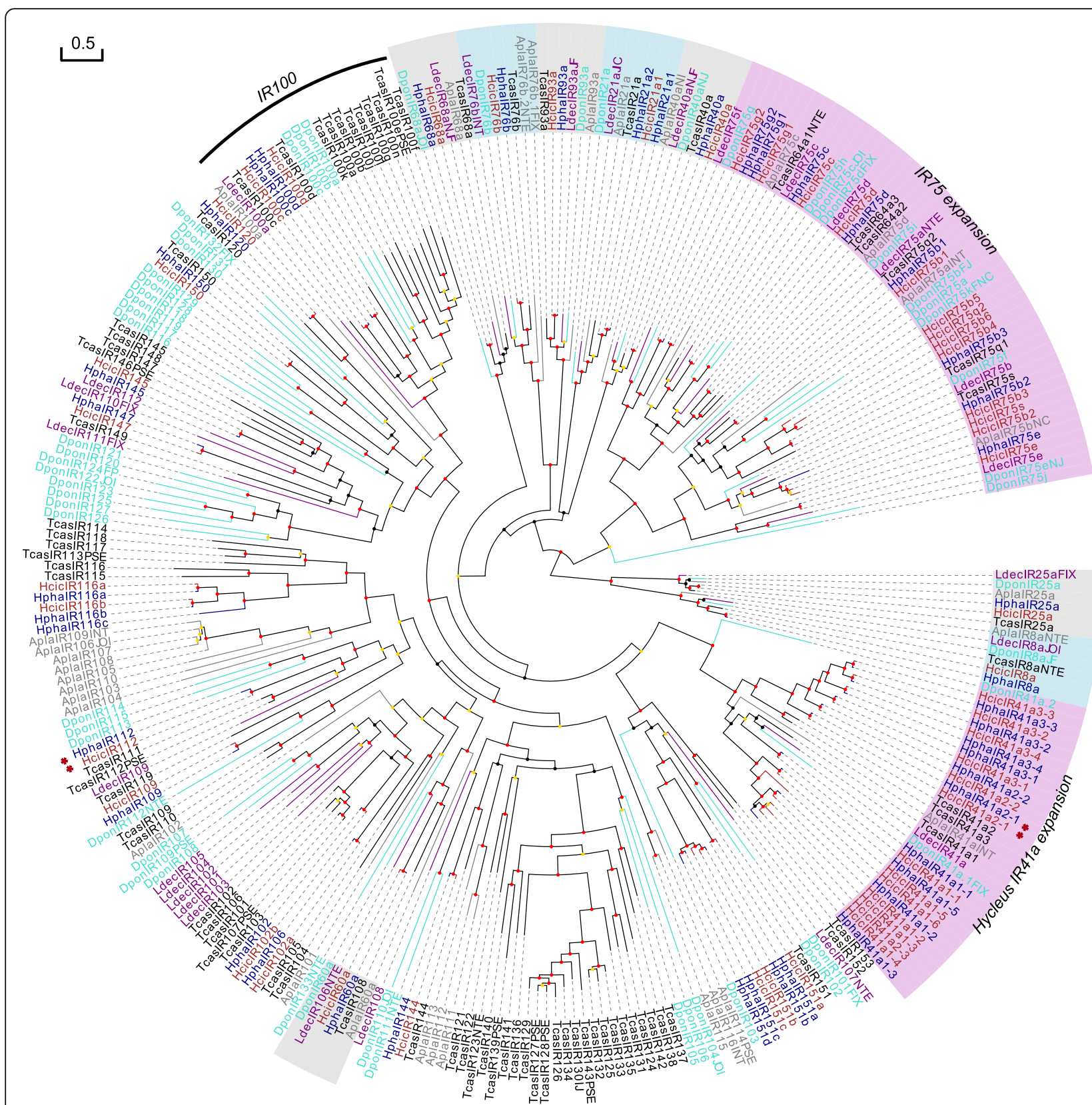

Fig. 3 Maximum Likelihood Phylogeny Based on the Protein Sequences of the Candidate IRs and iGluRs. Red: H. cichorii (Hcic); blue: H. phaleratus (Hpha); black: T. castaneum (Tcas); turquoise: D. ponderosae (Dpon); purple: L. decemlineata (Ldec) and grey: A. planipennis (Apla). The tree is based on MAFFT alignment, was constructed using RAxML, and was rooted with the conserved lineage of putative IR8a/25a clade. The coloured dots indicate nonparametric bootstrap support (\%): red: $\geq 80$, yellow: 50-79, and black: $<50$. The 'antennal IRs' are shaded with colours. Major Hycleusspecific expansion of 'IR41a' is evident, and the 'IR75' clade is expanded in both the Hycleus genus and bark beetle (plum shade). The red asterisk $\left.{ }^{*}\right)$ marks the positively selected gene pairs

and two distinct populations of the leaf beetle C. lapponica [7] have been partially assessed via RNA-seq. Hence, our study facilitates the analysis of chemosensory evolution among beetles, and we can now begin to discuss the question of how blister beetle diversification relates to the differentiation of these crucial gene families.
ORs are one of the key bridges between animals and their surroundings and play roles in the sensing of food, predators and potential mates. The OR family is the most widely studied among chemosensory receptors. Based on previous studies [27], nine groups of beetle ORs were identified in this study, and a similar pattern was observed in our phylogenetic tree. For example, 
Table 2 Number of Antennal IRs for the Seven Beetles. Major Hycleus-specific expansion of 'IR41a' is evident. 'IR75' expansion is observed in both the Hycleus genus and bark beetle. * One positively selected orthologue pair was identified in blister beetles

\begin{tabular}{|c|c|c|c|c|c|c|c|}
\hline & H. phaleratus & H. cichorii & T. castaneum [31] & D. ponderosae [28] & L. decemlineata [31] & A. planipennis [28] & A. glabripennis [32] \\
\hline IR25a & 1 & 1 & 1 & 1 & 1 & 1 & 1 \\
\hline IR8a & 1 & 1 & 1 & 1 & 1 & 1 & 1 \\
\hline IR75 & 8 & 13 & 5 & 11 & 6 & 4 & 8 \\
\hline IR40a & 1 & 1 & 1 & 1 & 1 & 1 & 1 \\
\hline$R 21 a$ & 2 & 1 & 1 & 1 & 1 & 1 & 1 \\
\hline IR68a & 1 & 1 & 1 & 1 & 1 & 1 & 1 \\
\hline IR41 $a^{*}$ & 10 & 13 & 3 & 2 & 1 & 1 & 1 \\
\hline IR76b & 1 & 1 & 1 & 1 & 1 & 2 & 1 \\
\hline IR93a & 1 & 1 & 1 & 1 & 1 & 1 & 1 \\
\hline IR60a & 1 & 1 & 1 & 1 & 1 & 1 & 1 \\
\hline Total & 27 & 34 & 16 & 21 & 15 & 14 & 17 \\
\hline
\end{tabular}

most ORs of $D$. ponderosae and A. planipennis were classified in Groups 7 and 2B, respectively, while Groups 4, 5B and 6 contained very few beetle ORs (Fig. 1). Notably, two expanded clades of ORs were identified based on our phylogenetic tree (red arcs in Groups 5A and 3; Fig. 1), and more than $1 / 2$ of the blister beetle ORs were included. Based on the phylogenetic tree, the divergence in these two clades is notably low, especially in Group
$5 \mathrm{~A}$, indicating that these ORs present similar sequences and emerged in a relatively short time. Moreover, the selection signals of 8 pairs of ORs were detected, and 5 pairs were from these two expanded clades, while 4 pairs and 1 pair were from Groups 5A and 3, respectively. These data suggest that the ORs in these expanded clades may have emerged along with the differentiation

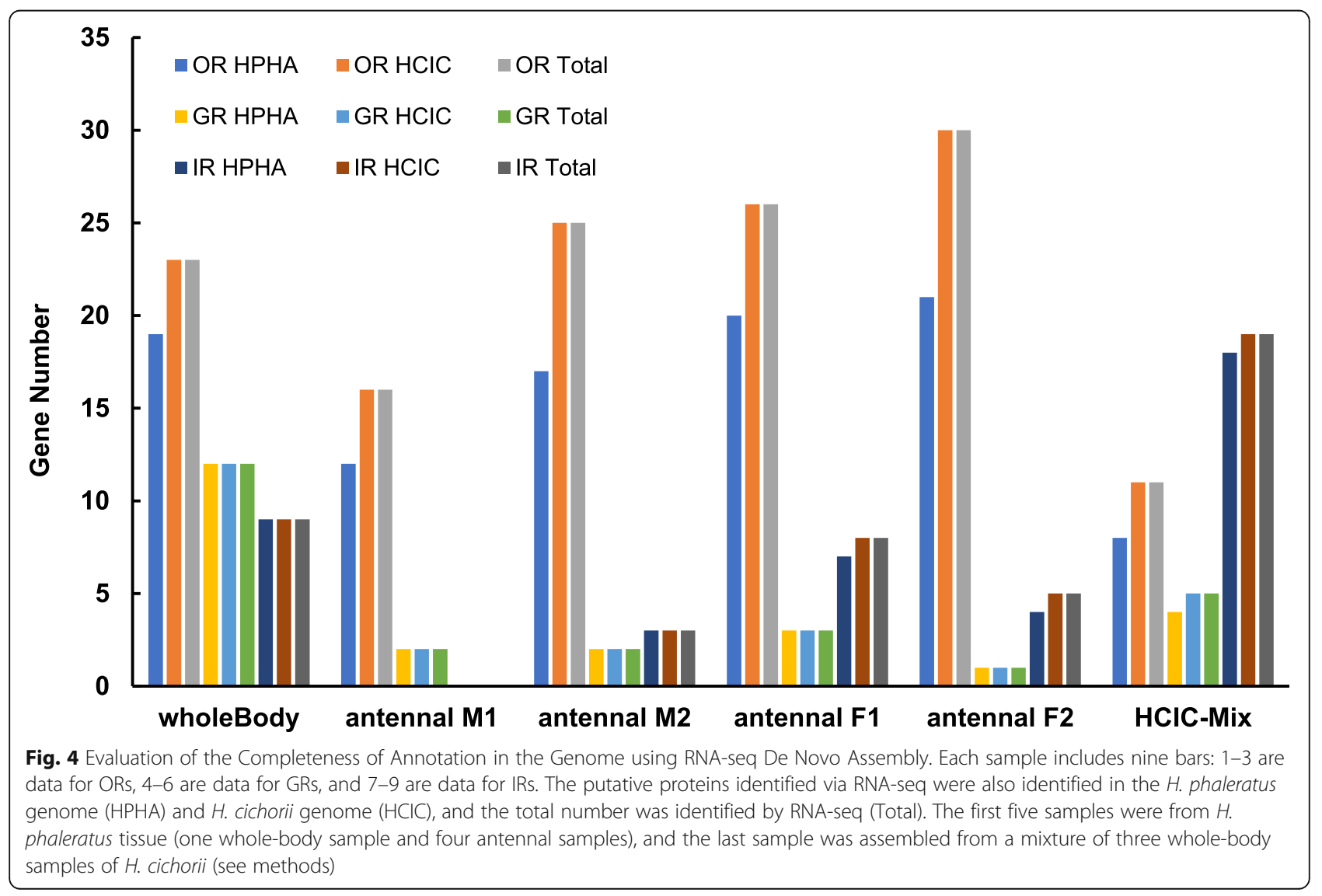


of both blister beetles, with speciation occurring approximately 23 million years ago [33].

Up to $67 \%$ more ORs were identified in $H$. cichorii than in its sister species (149 vs 89 ORs), and nearly all of these additional $\mathrm{H}$. cichorii ORs were clustered in two expanded clades (Groups 5A and 3). However, the ORs in the other clades showed nearly 1:1 orthologues in both blister beetles. On the other hand, our evaluation using transcriptome data showed that $\sim 17.4-30.0 \%$ of the ORs identified via RNA-seq were missing from the H. phaleratus genome (Fig. 4 and Table 1). This finding suggests that some members in these expanded clades were not present in the genome assembly, which is the main reason why fewer genes were identified in $H$. phaleratus than in its sibling beetle species. In $H$. cichorii, the ORs identified via RNA-seq were annotated in its genome (Fig. 4), making for more robust evolutionary analyses within Coleoptera. Additionally, compared to another recent report, a higher proportion of pseudogenes was annotated in the ORs (20.1 and 22.5\%), but the proportion was in the normal range since the value was close to that for the red flour beetle OR $(23.2 \%$ pseudogenes) [34].

GRs are divided into four groups based on the type of contact material, namely, $\mathrm{CO}_{2}$, sugar, and fructose receptors and putative bitter receptors. We identified three, eight and three pairs of $\mathrm{CO}_{2}$, sugar and fructose receptors, respectively, in both blister beetle species (Fig. 2). These groups are consistently highly conserved in insects, while the putative bitter receptors consistently show high divergence. However, the putative bitter GRs also exhibited good conservation among members of the Hycleus genus, with more than two-thirds of the $H$. phaleratus bitter GRs existing in 1:1 orthologue pairs. Similar to the ORs, two recently expanded clades were identified in the bitter GRs, with one gene showing evidence of positive selection. These data suggest that the GRs in these expanded clades may also be linked to the differentiation of both blister beetles. Although the gene number of putative bitter receptors is slightly greater in $H$. cichorii than in $H$. phaleratus (87 vs 72 ), this difference may be related to the difference in genome quality, with the scaffold N50 at $79 \mathrm{~kb}$ in $\mathrm{H}$. cichorii and $56 \mathrm{~kb}$ in $H$. phaleratus [33]. Moreover, nearly all the additional bitter GRs of $H$. cichorii were clustered in these expanded clades.

IRs are also membrane proteins that are closely related to iGluRs [29, 30]. We identified 45 and 50 IRs in $H$. phaleratus and $H$. cichorii, respectively. IRs can be divided into two groups: antennal IRs, which are consistently conserved among insect orders and function in olfaction in insects, and divergent IRs, which are highly divergent and expressed in peripheral and internal gustatory neurons, which indicates their involvement in taste and food assessment [29]. Ten clades of antennal IRs were identified in both blister beetles, and five of these subfamilies were conserved with a 1:1 orthologue in all seven beetles (Fig. 3 and Table 2). However, IR41a (10 and 13 in blister beetles vs 1-3 in others) was evidently expanded compared to the others (Fig. 3 and Table 2) and had the largest number identified in beetles to date. Based on the expansion reported in D. ponderosae [28], the expanded IR75 (8 and 13 in blister beetles and 11 in D. ponderosae [28] vs 4-8 in others) was also found in blister beetles. These data suggest that IR41a and IR75 underwent expansion in the Hycleus genus, which could have introduced new characteristics in blister beetles. One IR41a member (IR41a2-1) showed evidence of positive selection. To some degree, its expansion was accompanied by blister beetle speciation. We also identified IR60a (one copy in each beetle) in beetles based on our phylogenetic tree; this gene was misnamed in other beetles except for $D$. ponderosae and A. planipennis.

\section{Candidate chemosensory receptors linked to speciation}

Divergent chemosensory receptors linked to differences in the sensory tuning of sister species represent a response to divergent selection of chemosensory traits [1]; thus, these chemosensory genes may be associated with speciation and directly or indirectly reflect the ability to adapt to the environment. For instance, a comparison of the gene duplication of chemosensory gene families between the sister beetle species suggested some candidate genes that might be associated with the ability to adapt to changing environments. In the present study, two evident expansions of OR clades were identified in blister beetles. Five of eight OR pairs showing evidence of positive selection were included in these expanded clades. Similar to ORs, two recently expanded bitter GR clades and one that includes a member with signs of positive selection were identified. Moreover, IR41a was expanded specifically in both blister beetles, and one IR41a protein showed signs of positive selection. These data suggest that these ORs, GRs and IRs may be linked to reproductive isolation between the species. Unfortunately, some receptors of $H$. phaleratus were missing from the genome, and these should be identified and analysed in the future. On the other hand, we identified 13 pairs of orthologues that showed signs of positive selection, i.e., 8 ORs, 3 GRs and 2 IRs. Seven of these pairs also appeared in the cluster of gene duplication, i.e., 5 ORs, 1 GR (GR34) and one IR41a (Additional file 3). These two beetles have largely overlapping sympatric ranges in Southwest China. Based on our field survey experience, the $H$. phaleratus population has declined in recent years due to the destruction of its habitats by human activity, including capture by humans. In contrast, the $H$. cichorii population has not obviously declined in a 
similar manner. These gene duplications and amino acid variations may facilitate greater adaptation to environmental changes in $H$. cichorii than in $H$. phaleratus. Other methods, such as changes in gene expression, should be considered in future studies.

\section{Conclusions}

In the present study, we first performed comprehensive whole-genome identification of chemosensory receptor gene families in a pair of sister beetles (Meloidae: Hycleus). Several evolutionary events of blister beetles were revealed. For example, two expanded clades with positively selected proteins were identified in both ORs and bitter GRs, and two evident expansions of IR41a (blister beetle specific) and IR75 (Hycleus genus and D. ponderosae) were detected. This study will provide an important platform for future functional characterization of chemoreceptors and for uncovering the evolutionary history of blister beetles.

\section{Methods}

Identification of chemosensory receptors

The $H$. cichorii and $H$. phaleratus genome sequences were obtained from the NCBI database (BioProject accession number PRJNA390850) [33]. We identified OR, GR, and IR genes using the following steps. First, we constructed a protein reference database of each family using published proteins from Tribolium castaneum, D. ponderosae, Anoplophora glabripennis, A. planipennis and Leptinotarsa decemlineata [27-29, 31, 32, 35]. Second, tBLASTn (version 2.2.25) was used to search the exons in both genomes with an e-value cut-off of 1e-5. Third, we first linked the alignment hits into candidate gene loci exon by exon with an in-house Perl script. We then extracted genomic sequences of candidate loci together with 2-kb flanking sequences and used GeneWise [36] to determine the gene models. Next, putative proteins were manually compared with other genes of the same family and RNA-seq data (NCBI SRA database, SRA accession number SRR5408725, SRR5408726, SRR5757329, SRR5757330, SRR9702113, SRR9702114, SRR9702115, and SRR9702116 for $H$. phaleratus and SRR4436644, SRR4436645, and SRR1996329 for $H$. cichorii), and only the best gene model/splice isoform with the highest score provided by GeneWise was retained. For incomplete genes, putative proteins with at least half the length of the reference protein and a size larger than 190 amino acids were retained. Genes with premature stop codons or frameshifts were defined as pseudogenes. The premature stop codons were replaced by $\mathrm{X}$ in the translated sequence. Finally, we assessed functional annotations in public protein databases (NCBI nonredundant protein and Swiss-Prot) using BLASTp and removed candidate genes with inconsistent family annotations. To avoid missing some putative genes, we performed two rounds of identification using the above pipeline. In the second round, the putative proteins identified in the first round were also included as queries for tBLASTn and GeneWise.

To verify whether some chemosensory receptor genes were missing in the annotation process and in the genome, we de novo assembled RNA-seq data and counted the number of candidates. The RNA-seq data for $H$. phaleratus (whole body and antennae) were obtained from the NCBI SRA database (SRA accession number SRR5408725, SRR5408726, SRR5757329, SRR5757330, SRR9702113, SRR9702114, SRR9702115, and SRR9702116), and the data for $H$. cichorii were also obtained from the NCBI SRA database (SRA accession numbers: SRR4436644, SRR4436645, and SRR1996329). After removing adaptor commination and low-quality reads, Trinity (version v2.0.6; http://trinityrnaseq.sourceforge.net/) [37] was applied with the following parameters: group_pairs_distance $=300$; min_contig_length $=$ 150; min_kmer_cov $=3$; and min_glue $=3$. Then, the assembled sequences were mapped onto the known reference protein (the same proteins as in the identification step) and genome sequences with BLAT [38], and the candidates were counted, the homologous regions of which contained at least 120 (ORs and GRs) or 190 (IRs) amino acids.

\section{Phylogenetic trees}

To identify orthologous pairs between the two sister species and analyse the relationships of Hycleus chemosensory receptors with those of other insects, ML trees for each family were constructed using the amino acid sequences derived from the putative CDSs and the published proteins of other species, including T. castaneum, D. ponderosae, A. planipennis and L. decemlineata (Additional file 4) [28, 31, 32, 35]. First, multiple-sequence alignment was performed by MAFFT (version 7.407) [39] using the amino acid sequences corresponding to the chemosensory receptors of these species. Then, RAxML (v8.2.12) [40] was used to construct phylogenetic trees of the chemosensory receptors with the best models (JTT for IRs, JTT + G + F for ORs and GRs), and node support was assessed using a bootstrap procedure based on 200 replicates. The phylogenetic trees were visualized with EvolView (http://evolgenius.info/evolview) [41]. To facilitate a more robust phylogenetic analysis, partial ORs with fewer than 230 amino acids were excluded from the phylogenetic analysis. Due to the highly divergent nature of GRs, amino acid sequences with fewer than 250 amino acids were also excluded [28]. The OR tree was rooted with the conserved lineage of odorant receptor coreceptor (Orco) proteins, the GR tree was rooted with the conserved lineage of putative sugar 
receptors, and the IR tree was rooted with the conserved lineage of putative IR8a/25a proteins.

\section{Selection analysis}

According to the phylogenetic trees, we selected the orthologous gene pairs between these two Hycleus species for selection analysis. The paired orthologues of the protein sequences were aligned using MAFFT (version 7.407) [39]. Then, the alignment information of the protein sequences was used as a guide for nucleic acid sequence alignment. Finally, analyses of nonsynonymous (KA) and synonymous (KS) substitutions were performed by codeml in the PAML package (v4.9) [42] using the site model (M8 [beta and $\omega>1$ ] vs M7 [beta]). To test whether each site in an orthologue pair underwent significant selection, the likelihood ratio was estimated based on the M8 model and M7 model. The LRT statistic uses the formula $\Delta=2(\ln (\mathrm{M} 8)-\ln (\mathrm{M} 7))$, with $\Delta$ approximating a chi-square distribution with one degree of freedom [26]. The putative selected genes had at least one selected site and passed the chi-square test using a $P$-value cut-off of 0.01 . All the selected sites had to pass both test methods (Bayes empirical Bayes [BEB] and naive empirical Bayes [NEB], provided by codeml software) using a probability cut-off of 0.95 , and the quality of the alignment was manually assessed.

\section{Supplementary information}

Supplementary information accompanies this paper at https://doi.org/10. 1186/s12864-020-06974-4.

Additional file 1. Amino acid and nucleotide sequences of ORs, IRs and GRs identified in this work.

Additional file 2. Gene location information (gff).

Additional file 3. All positively selected gene pairs between the two blister beetle species.

Additional file 4. The protein set used in phylogenetic tree analysis.

\section{Abbreviations}

GR: Gustatory receptor; OR: Odorant receptor; IR: Ionotropic receptor; iGluR: Ionotropic glutamate receptor; ML: Maximum likelihood; CDS: Coding sequence; NGS: Next-generation sequencing; BUSCO: Benchmarking Universal Single-Copy Orthologs

\section{Acknowledgements}

We thank Dr. Wanshun Li (Southwest University) for providing valuable advice and discussion. We also thank the three anonymous reviewers for their valuable suggestions and comments. In addition, we thank the editor of BMC Genomics for improving the writing.

\section{Authors' contributions}

$X C$ and $Y W$ designed the projects. $Y W$ and $Y L$ collected the insects and extracted the total RNA samples. YW performed the data analysis and produced the figures and tables. YW and XC wrote the manuscript. All authors read and approved the final manuscript.

\section{Funding}

The study was supported by the open research fund of the Key Laboratory Of Environmental Pollution Monitoring and Disease Control, Ministry of Education (Qianjiaohe KY Zi [2019] 045), the project of Young Scientific and
Technical Talents Growth, Guizhou Provincial Education Department (Qianjiaohe KY Zi [2018] 177), the National Natural Science Foundation of China (no. 81460576), the Programme of Excellent Innovation Talents, Guizhou Province (no. 20154021), and the International Cooperation Base for Insect Evolutionary Biology and Pest Control (no. 20165802). The funding bodies played no role in the design of the study; in the collection, analysis, and interpretation of the data; or in the writing of the manuscript.

\section{Availability of data and materials}

All data generated by this study are included in this published article and its supplementary information file $1-3$. The proteins of $T$. castaneum, $D$. ponderosae, A. glabripennis, A. planipennis, and L. decemlineata were retrieved from the previous publications, used as BLAST query $[27-29,31,32,35]$ and for phylogenetic tree were summarized in Additional file 4. The reference genome sequences of $\mathrm{H}$. cichorii and $\mathrm{H}$. phaleratus were obtained from the NCBI database (BioProject accession number PRJNA390850). The RNA-seq data were used for annotation and check from NCBI SRA database (SRA accession number SRR5408725, SRR5408726, SRR5757329, SRR5757330, SRR9702113, SRR9702114, SRR9702115, and SRR9702116 for H. phaleratus; and the SRR4436644, SRR4436645, and SRR1996329 for H. cichorii).

\section{Ethics approval and consent to participate}

Not applicable.

\section{Consent for publication}

Not applicable.

\section{Competing interests}

The authors declare that they have no competing interests.

\section{Author details}

'School of Basic Medical Sciences, Guizhou Medical University, Guiyang, Guizhou, P.R. China 550025. ${ }^{2}$ Key Laboratory of Environmental Pollution Monitoring and Disease Control, Ministry of Education, Guizhou Medical University, Guiyang, Guizhou, P.R. China 550025. 'Institute of Entomology/ Special Key Laboratory for Development and Utilization of Insect Resources, Guizhou University, Guiyang, Guizhou, P.R. China 550025.

Received: 14 February 2020 Accepted: 6 August 2020

Published online: 26 August 2020

\section{References}

1. Smadja C, Butlin RK. On the scent of speciation: the chemosensory system and its role in premating isolation. Heredity. 2009:102:77-97.

2. McBride CS. Rapid evolution of smell and taste receptor genes during host specialization in Drosophila sechellia. Proc Natl Acad Sci U S A. 2007;104: 4996-5001.

3. Matsuo T, Sugaya S, Yasukawa J, Aigaki T, Fuyama Y. Odorant-binding proteins OBP57d and OBP57e affect taste perception and host-plant preference in Drosophila sechellia. PLoS Biol. 2007;5:e118.

4. Shiao MS, Chang JM, Fan WL, Lu MY, Notredame C, Fang S, et al. Expression divergence of chemosensory genes between Drosophila sechellia and its sibling species and its implications for host shift. Genome Biol Evol. 2015;7: 2843-58.

5. Smadja CM, Canback B, Vitalis R, Gautier M, Ferrari J, Zhou JJ, et al. Largescale candidate gene scan reveals the role of chemoreceptor genes in host plant specialization and speciation in the pea aphid. Evolution. 2012;66: 2723-38.

6. Zhang B, Zhang W, Nie RE, Li WZ, Segraves KA, Yang XK, et al. Comparative transcriptome analysis of chemosensory genes in two sister leaf beetles provides insights into chemosensory speciation. Insect Biochem Mol Biol. 2016;79:108-18

7. Wang $D$, Pentzold $S$, Kunert $M$, et al. A subset of chemosensory genes differs between two populations of a specialized leaf beetle after host plant shift. Ecol Evol. 2018;8:8055-75. https://doi.org/10.1002/ece3.4246.

8. Kaupp UB. Olfactory signalling in vertebrates and insects: differences and commonalities. Nat Rev Neurosci. 2010;11:188-200.

9. Touhara K, Vosshall LB. Sensing odorants and pheromones with chemosensory receptors. Annu Rev Physiol. 2009;71:307-32.

10. Carey AF, Wang G, Su C-Y, Zwiebel LJ, Carlson JR. Odorant reception in the malaria mosquito Anopheles gambiae. Nature. 2010;464:66-71. 
11. Hallem EA, Carlson JR. Coding of odors by a receptor repertoire. Cell. 2006; 125:143-60.

12. Stensmyr MC, Dweck HK, Farhan A, Ibba I, Strutz A, Mukunda L, et al. A conserved dedicated olfactory circuit for detecting harmful microbes in Drosophila. Cell. 2012;151:1345-57.

13. Kwon JY, Dahanukar A, Weiss LA, Carlson JR. The molecular basis of $\mathrm{CO}_{2}$ reception in Drosophila. Proc Natl Acad Sci U S A. 2007;104:3574-8.

14. Robertson HM, Kent LB. Evolution of the gene lineage encoding the carbon dioxide receptor in insects. J Insect Sci. 2009;9:19.

15. Vosshall LB, Stocker RF. Molecular architecture of smell and taste in Drosophila. Annu Rev Neurosci. 2007;30:505-33.

16. Benton R, Vannice KS, Gomez-Diaz C, Vosshall LB. Variant ionotropic glutamate receptors as chemosensory receptors in Drosophila. Cell. 2009; 136:149-62.

17. Abuin $L$, Bargeton $B$, Ulbrich MH, Isacoff EY, Kellenberger $S$, Benton $R$. Functional architecture of olfactory ionotropic glutamate receptors. Neuron. 2011;69:44-60

18. Till JS, Majmudar BN. Cantharidin poisoning. South Med J. 1981;74(4):444-7.

19. Day RM, Harbord M, Forbes A, Segal AW. Cantharidin blisters: a technique for investigating leukocyte trafficking and cytokine production at sites of inflammation in humans. J Immunol Methods. 2001;257:213-20.

20. Silverberg NB, Sidbury R, Mancini AJ. Childhood molluscum contagiosum: experience with cantharidin therapy in 300 patients. J Am Acad Dermatol. 2000:43:503-7.

21. Liu D, Chen Z. The effects of cantharidin and cantharidin derivates on tumor cells. Anti Cancer Agents Med Chem. 2009;9:392-6.

22. Zhang $W, M a Y Z$, Song $L$, et al. Effect of cantharidins in chemotherapy for hepatoma: a retrospective cohort study. Am J Chin Med. 2014;42(03):561-7.

23. Kadioglu O, Kermani NS, Kelter G, et al. Pharmacogenomics of cantharidin in tumor cells. Biochem Pharmacol. 2014;87(3):399-409.

24. Editorial Board of Pharmacopoeia of the People's Republic of China. Pharmacopoeia of the People's republic of China. Beijing: Chemical Industry Press; 2005.

25. Zhang S, Zhang Z, Wang H, Kong X. Antennal transcriptome analysis and comparison of olfactory genes in two sympatric defoliators, Dendrolimus houi and Dendrolimus kikuchii (Lepidoptera: Lasiocampidae). Insect Biochem Mol Biol. 2014;52:69-81.

26. Brand P, Ramírez SR, Leese F, Ouezada-Euan JJ, Tollrian R, Eltz T. Rapid evolution of chemosensory receptor genes in a pair of sibling species of orchid bees (Apidae: Euglossini). BMC Evol Biol. 2015:15:176.

27. Mitchell RF, Schneider TM, Schwartz AM, Andersson MN, McKenna DD. The diversity and evolution of odorant receptors in beetles (Coleoptera). Insect Mol Biol. 2019. https://doi.org/10.1111/imb.12611.

28. Andersson MN, Keeling Cl, Mitchell RF. Genomic content of chemosensory genes correlates with host range in wood-boring beetles (Dendroctonus ponderosae, Agrilus planipennis, and Anoplophora glabripennis). BMC Genomics. 2019;20:690. https://doi.org/10.1186/s12864-019-6054-x.

29. Croset V, Rytz R, Cummins SF, Budd A, Brawand D, Kaessmann H, et al. Ancient protostome origin of chemosensory ionotropic glutamate receptors and the evolution of insect taste and olfaction. PLoS Genet. 2010;6: e1001064.

30. Rytz R, Croset V, Benton R. Ionotropic receptors (IRs): chemosensory ionotropic glutamate receptors in Drosophila and beyond. Insect Biochem Mol Biol. 2013;43:888-97. https://doi.org/10.1016/j.ibmb.2013.02.007.

31. Schoville SD, Chen YH, Andersson MN, Benoit JB, Bhandari A, Bowsher JH, et al. A model species for agricultural pest genomics: the genome of the Colorado potato beetle, Leptinotarsa decemlineata (Coleoptera: Chrysomelidae). Sci Rep. 2018;8:1931.

32. McKenna DD, Scully ED, Pauchet Y, Hoover K, Kirsch R, Geib SM, et al. Genome of the Asian longhorned beetle (Anoplophora glabripennis), a globally significant invasive species, reveals key functional and evolutionary innovations at the beetle-plant interface. Genome Biol. 2016;17:227.

33. Wu Y, Li J, Chen X. Draft genomes of two blister beetles Hycleus cichorii and Hycleus phaleratus. Gigascience. 2018;7:1-7. https://doi.org/10.1093/ gigascience/giy006.

34. Engsontia P, Sanderson AP, Cobb M, Walden KK, Robertson HM, Brown S. The red flour beetle's large nose: an expanded odorant receptor gene family in Tribolium castaneum. Insect Biochem Mol Biol. 2008:38:387-97.

35. Dippel S, Kollmann M, Oberhofer G, Montino A, Knoll C, Krala M, et al. Morphological and transcriptomic analysis of a beetle chemosensory system reveals a gnathal olfactory center. BMC Biol. 2016;14:90
36. Birney E, Clamp M, Durbin R. GeneWise and Genomewise. Genome Res. 2004;14:88-95.

37. Grabherr MG, Haas BJ, Yassour M, Levin JZ, Thompson DA, Amit I, Adiconis X, Fan L, Raychowdhury R, Zeng Q, Chen Z, Mauceli E, Hacohen N, Gnirke A, Rhind N, di Palma F, Birren BW, Nusbaum C, Lindblad-Toh K, Friedman N, Regev A. Full-length transcriptome assembly from RNA-Seq data without a reference genome. Nat Biotech. 2011;29:644-52.

38. Kent W. James. BLAT--the BLAST-like alignment tool. Genome Res. 2002; 12(4):656-64. https://doi.org/10.1101/gr.229202.

39. Katoh K, Standley DM. MAFFT multiple sequence alignment software version 7: improvements in performance and usability. Mol Biol Evol. 2013; 30:772-80.

40. Guindon S, Dufayard JF, Lefort V, et al. New algorithms and methods to estimate maximum-likelihood phylogenies: assessing the performance of PhyML 3.0. Syst Biol. 2010:59:307-21.

41. Subramanian B, Gao S, Lercher MJ, Hu S, Chen W-H. Evolview v3: a webserver for visualization, annotation, and management of phylogenetic trees. Nucleic Acids Res. 2019;47(Issue W1):W270-5. https://doi.org/10.1093/ nar/gkz357.

42. Yang Z. PAML 4: phylogenetic analysis by maximum likelihood. Mol Biol Evol. 2007;24:1586-91.

\section{Publisher's Note}

Springer Nature remains neutral with regard to jurisdictional claims in published maps and institutional affiliations.
Ready to submit your research? Choose BMC and benefit from:

- fast, convenient online submission

- thorough peer review by experienced researchers in your field

- rapid publication on acceptance

- support for research data, including large and complex data types

- gold Open Access which fosters wider collaboration and increased citations

- maximum visibility for your research: over $100 \mathrm{M}$ website views per year

At BMC, research is always in progress.

Learn more biomedcentral.com/submissions 